Cochrane erstellt systematische Übersichtsarbeiten zu medizinischen Forschungsfragen und schafft damit eine zuverlässige Grundlage für die evidenzbasierte Medizin. Seit 2016 haben alle in der Schweiz wohnhaften Personen kostenlosen Zugang zur Cochrane Library. Im Rahmen einer vierteiligen Artikelserie stellen wir die Cochrane Library vor. In diesem vierten Artikel befasst sich Dr. med. Erik von Elm, Direktor von Cochrane Schweiz, mit den Chancen und Herausforderungen bei der Nutzung der Cochrane Library.

Dr. med. Christoph Bosshard, Vizepräsident der FMH, Departementsverantwortlicher Daten, Demographie und Qualität

\title{
Die Cochrane Library im Fokus
}

\author{
Interview: Dr. sc. Stefanie Hostettler
}

Dr. von Elm, Sie sind Direktor von Cochrane Schweiz. Welchen Stellenwert hat Cochrane in der Forschung, und was ist der Anreiz, bei Cochrane mitzuarbeiten? Cochrane setzt sich seit über 25 Jahren dafür ein, dass die Ergebnisse der Forschung systematisch gesucht, bewertet und zusammengefasst werden. Unsere Arbeit ermöglicht es, dass die Evidenz dahin kommt, wo sie am dringendsten gebraucht wird: bei den Gesundheitsentscheidungen auf allen Ebenen. Das motiviert mich genauso wie einige tausend andere CochraneMitglieder weltweit, zur Arbeit dieses Netzwerks beizutragen.

\section{Was hat sich seit der Einführung der Cochrane Library verändert?}

Von Anfang an waren der Ausbau und die Verbesserung der Cochrane Library mit ihrer Sammlung systematischer Reviews Kernstück der Arbeit. Cochrane selbst hat sich seit den Anfängen in den 1990er Jahren von einem losen Netzwerk gleichgesinnter Akademiker zu einer internationalen Organisation gewandelt, in der ausser Forschern auch Personen mit anderen Blickwinkeln wie z.B.

Cochrane setzt sich seit über 25 Jahren dafür ein, dass die Ergebnisse der Forschung systematisch gesucht, bewertet und zusammengefasst werden.

Patienten mitwirken. Die Cochrane Library hat dazu beigetragen, dass die wissenschaftliche Evidenz einen wesentlichen Einfluss auf die ärztliche Tätigkeit hat.

\section{Für welche Zielgruppe ist die Cochrane Library gemacht? Wie erreicht ihr die Zielgruppen?}

Die wichtigste Zielgruppe sind Fachpersonen im Gesundheitswesen. Mittlerweile stehen auch für Laien etliche tausend Zusammenfassungen nicht nur auf

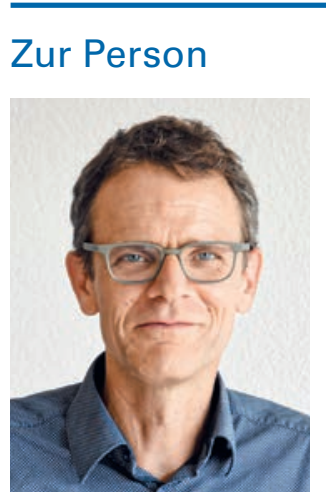

\section{Dr. med. Erik von Elm}

$M S c, F M H$ Prävention und Gesundheitswesen

Erik von Elm ist Mitbegründer und Direktor von Cochrane Schweiz. Nach dem Medizinstudium und der Promotion in Deutschland und Frankreich hat er an der London School of Hygiene and Tropical Medicine den Master-Abschluss in Epidemiologie und in der Schweiz den Facharzt in Prävention und Gesundheitswesen erworben.

Er ist Mitinitiator und Autor von Initiativen zur Verbesserung der Qualität von wissenschaftlichen Artikeln, wie z.B. dem STROBEStatement für Berichte von Beobachtungsstudien, und Gutachter für medizinische Journale. Als wissenschaftlicher Oberarzt ist er in Forschung und Lehre am Centre universitaire de médecine générale et santé publique (Unisanté) in Lausanne tätig und lehrt u.a. zu Fragen der Methodik, der Forschung in klinischer Medizin und Public Health. Er vertritt als gewähltes Mitglied die nationalen Zentren im Cochrane Council.

Englisch, sondern auch in vereinfachter Sprache auf Deutsch, Französisch und in zahlreichen anderen Sprachen zur Verfügung. Damit wird die gesammelte Evidenz auch medizinischen Laien zugänglich gemacht. Zwischen Ärztin und Patient kann so eine Diskussion stattfinden, in der nicht nur eine Seite die Informationen hat.

\section{Was hat die Finanzierung der Nationallizenz 2016 durch die SAMW, das BAG und die Universitäts- bibliotheken gebracht?}

Das war ein wichtiger Schritt, um den uns die Cochrane-Kolleginnen und -Kollegen in einigen Län- 
dern beneiden! Die Zugangszahlen haben sich vom ersten Tag an verdoppelt und sind konstant hoch geblieben. Wir hoffen, dass in Zukunft noch mehr Menschen mit Gesundheitsfragen dieses Angebot nutzen werden.

\section{Welchen Mehrwert erfahren Ärztinnen, Ärzte und Gesundheitsfachpersonen durch die Nutzung der Cochrane Library?}

Der grösste Mehrwert ist wohl die Zeitersparnis, da man sich nicht durch einzelne, zum Teil widersprüchliche Fachartikel kämpfen muss, sondern deren Ergebnisse in einer Übersichtsarbeit geliefert bekommt. Dabei hilft auch das Format «Clinical Answers» - quasi als Schnellzugang zur Evidenz.

Der grösste Mehrwert ist wohl die Zeitersparnis, da man die Ergebnisse in einer Übersichtsarbeit geliefert bekommt.

Hausarzt Dr. med. Bruno Kissling zeigt in seinem Artikel auf, dass hausarztspezifische Fragestellungen oft komplex sind und es meist mehrere medizinische Probleme zu behandeln gibt. Wie kann die Cochrane Library die Ärzteschaft bei diesen hausarztspezifischen Gegebenheiten unterstützen?

Für die Analyse der Situation eines Patienten braucht es nach wie vor die Erfahrung der Fachperson, die Gesundheitsprobleme einordnen und gewichten kann. Die Komplexität z.B. durch die Multimorbidität wird bisher in keiner medizinischen Informationsquelle zufriedenstellend abgebildet. Auch der Einsatz von Technologien wie der künstlichen Intelligenz steht da immer noch ganz am Anfang. Wenn aber eine Reihe von konkreten Fragen auf dem Tisch liegt, kann mit Hilfe der Cochrane Library geschaut werden, ob es z.B. für eine Medikation überhaupt einen ausreichenden Nachweis der Wirksamkeit gibt. Wenn das nicht der Fall ist, können unnötige oder sogar riskante Therapien vielleicht sogar weggelassen werden.

Dr. med. Bruno Kissling und Professor Dr. med. Fabian Krause berichten in den Ausgaben Nr. 46 und 47 der «Schweizerischen Ärztezeitung», dass die Suche in der Cochrane Library teilweise keine oder nicht aktuelle Ergebnisse zeigt, was dazu führen kann, dass die Cochrane Library nicht genutzt wird. Was raten Sie, und sind Massnahmen zur Optimierung des Angebots geplant?

Ein einzelnes Stichwort als Suchbegriff führt selten direkt zum gewünschten Ergebnis. Mit standardisierten Begriffen wie den MeSH (Medical Subject Heading
Terms), die auch in der Datenbank PubMed verwendet werden, geht das schon besser. Das Aktualisieren der vorhandenen Cochrane Reviews ist sehr aufwendig, denn laufend kommen neue Studienergebnisse dazu. Cochrane versucht hier, Prioritäten zu setzen und den Autorengruppen Unterstützung anzubieten, so dass Reviews von hoher Relevanz schneller verfasst oder aktualisiert werden. Mittlerweile gibt es die ersten sogenannten "Living Systematic Reviews», in welche neue Studien fortlaufend eingeschlossen werden. Das bietet bisher keine andere Datenbank.

\section{Was sind Ihres Erachtens die Vorteile der Cochrane Reviews gegenüber den Guidelines?}

Im besten Fall sind ein oder mehrere Cochrane Reviews Grundlage für eine evidenzbasierte Guideline. Das ist bei vielen WHO-Guidelines heute so. Wer sich als Arzt oder Ärztin in seiner oder ihrer Tätigkeit auf solche Guidelines abstützt, liegt sicher nicht falsch. Es sollte aber kein "Entweder-oder» sein. Mit einer Reihe von Einzelempfehlungen in einer Guideline wird ein anderes Informationsbedürfnis gedeckt als mit einem systematischen Review, der - möglichst transparent darlegt, welche wissenschaftliche Evidenz wir aktuell haben.

\section{Wie unterscheiden sich Cochrane Reviews von HTA-Berichten?}

In einem klassischen Cochrane Review zu einer Behandlungsform wird die international vorhandene Evidenz zum klinischen Nutzen und Schaden systematisch aufbereitet. Das Gleiche gilt für diagnostische oder präventive Massnahmen. Diese Evidenz wird in HTA-Berichten ergänzt durch eine gesundheitsökonomische, ethische und rechtliche Analyse. Dazu kommt

Die Schlussfolgerungen von HTA-Berichten sind nur begrenzt auf andere Länder übertragbar, bei Cochrane Reviews ist das viel besser möglich.

die Einordnung der Fakten durch ein unabhängiges Gremium. Die Schlussfolgerungen von HTA-Berichten sind nur begrenzt auf andere Länder übertragbar, während dies bei Cochrane Reviews viel besser möglich ist.

Cochrane Reviews basieren häufig auf einem selektierten Patientenkollektiv. Inwiefern sind die Ergebnisse gültig für Patienten, die in der untersuchten Gruppe untervertreten sind wie z.B. Multimorbide, Frauen oder ältere Menschen?

Ein Cochrane Review basiert auf den Daten der eingeschlossenen Studien. Wenn diese nur eine selektierte 
Untergruppe widerspiegeln, schränkt das auch die Aussagekraft des Reviews ein. Oft unterscheiden sich aber die Studien bezüglich der Einschlusskriterien. Man spricht dann von Heterogenität. Einerseits ist das für das Kombinieren von Studiendaten in einer Metaanalyse problematisch. Anderseits erlaubt es aber oft eine Aussage darüber, ob ein Ergebnis (z.B. die Über-

\section{Ein Cochrane Review basiert auf den Daten der eingeschlossenen Studien.}

legenheit einer Therapie) verallgemeinert werden kann, also nicht nur für eine ausgewählte Gruppe von «Idealpatienten» gilt.

\section{Die patientenzentrierte Medizin gewinnt an Bedeutung; inwiefern werden die Bedürfnisse und Sichtweisen der Patienten und Patientinnen bei Cochrane berücksichtigt?}

Das geschieht auf mehreren Ebenen: Bei der Auswahl von Zielkriterien (sog. Outcomes oder Endpunkten), an denen die Wirksamkeit einer Therapie gemessen wird, stehen in Cochrane Reviews die für die Patienten relevanten an erster Stelle. Das heisst, dass Kriterien wie Funktionsfähigkeit, Schmerz oder Lebensqualität im Vordergrund stehen, selbst wenn in den eingeschlossenen Studien die Gruppen für eine Vielzahl von Laborparametern verglichen wurden.

Eine weitere Ebene ist der Einbezug von Patienten und Patientinnen in die Arbeit von Cochrane. Dafür gibt es ein eigenes "Cochrane Consumer Network», das die Patientenperspektive zum Beispiel dann einbringt, wenn es darum geht, in der Arbeit der ReviewGruppen thematische Prioritäten zu setzen.
Wo sehen Sie die Rolle der Politik im Zusammenhang mit Cochrane? Und welche Aufgabe können die

Stakeholder im Gesundheitswesen im Zusammenhang mit der Förderung der Cochrane Library übernehmen?

Es wäre wünschenswert, wenn in den politischen Prozessen die verfügbare wissenschaftliche Evidenz als Argument höher gewichtet würde. Beim Thema Klimawandel gehen mittlerweile Schüler auf die Strassen, um uns Erwachsene zur Evidenzbasierung zu mahnen: dass wir den Aussagen der Wissenschaftler endlich Taten folgen lassen müssen. Das hätte ich mir vor 20 Jahren nicht träumen lassen!

Ob bei gesundheitspolitischen Fragen die Evidenz nun von Cochrane kommt oder aus anderen vertrauenswürdigen Quellen, ist zweitrangig. Wichtig ist, dass sie bei einer Entscheidung ausreichend berücksichtig wird. Das heisst nicht, dass andere Argumente wie die Präferenzen der Betroffenen ausgeblendet werden sollen. Auf die hohe Qualität in unserem Gesundheitssystem können wir zu Recht stolz sein, aber nicht, wenn wir es uns weiterhin leisten, Massnahmen ohne ausreichenden Wirkungsnachweis zu finanzieren, und das ganze System irgendwann unbezahlbar wird. Mit der Cochrane Library steht in der

\section{Mit der Cochrane Library steht in der Schweiz} jedem eine unabhängige evidenzbasierte Informationsquelle frei zur Verfügung.

Schweiz jedem eine unabhängige evidenzbasierte Informationsquelle frei zur Verfügung. Ich hoffe, dass diese in Zukunft finanziell sogar noch breiter abgestützt werden kann.

\section{Über Cochrane}

Cochrane ist ein globales, unabhängiges Netzwerk von Wissenschaftlerinnen und Wissenschaftlern, Gesundheitsfachleuten, Patientinnen und Patienten und anderen Personen mit gesundheitsbezogenen Interessen. Zur Arbeit von Cochrane tragen ca. 11000 Mitglieder und über 68000 Unterstützer aus über 130 Ländern bei. Auf wissenschaftliche Methoden gestützt, stellen sie Gesundheitsinformationen bereit, die zuverlässig und frei von kommerziellen Sponsorengeldern sind. Jeder Cochrane Review widmet sich einer klar formulierten Fragestellung. Zu deren Beantwortung sucht ein Autorenteam alle vorhandenen Originalstudien, welche die zuvor definierten Einschlusskriterien erfüllen.
Anschliessend werden die eingeschlossenen Studien bewertet, um zu bestimmen, ob es zuverlässige Evidenz zu einer bestimm ten Behandlung, Diagnostik oder vorbeugenden Massnahmen gibt. Wenn möglich, werden die Einzelergebnisse in einer Metaanalyse kombiniert. Cochrane Reviews werden vor der Veröffentlichung im Peer-Review-Verfahren von Fachexperten begutachtet. Über 8000 Reviews sind bisher auf www.cochranelibrary.com zugänglich. Neben dem oft sehr ausführlichen Volltext stehen verschiedene Kurzformate zur Verfügung. Für die klinische Praxis besonders relevante Reviews werden unter "Clinical Answers" in einem Frage-Antwort-Format dargestellt. 